\title{
Diagnostic accuracy and usability of home calprotectin
}

testing

\section{Royal Devon and Exeter WHS \\ NHS Foundation Trust}

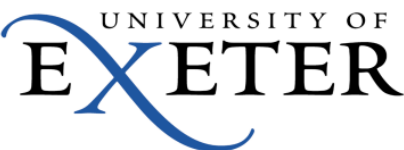
A. Thomas, M. Clarke, V. Cairnes, G. Walker, H. Green, J. Goodhand, T. Ahmad, N. Kennedy

\section{Background}

Faecal calprotectin (FC) - reliable and non-invasive stool biomarker used to monitor inflammation in inflammatory bowel disease (IBD)

$>$ Routinely performed by ELISA (time consuming and costly).

$>$ QuantonCal is an immunologic rapid test that measures calprotectin via a lateral flow device to a smart phone. Designed for use by patient at home

Delivers result within 15 minutes.

We sought to compare the accuracy, usability and patient perception of QuantonCal against ELISA.

\section{Methods}

Patients attending outpatient clinic at the Royal Devon and Exeter Hospital, UK approached

$>$ Inclusion criteria: owning a smartphone and need for disease activity/inflammation assessment

Patients collected a stool sample to perform QuantonCal and submitted a sample for ELISA analysis to assess correlation.

Patients also invited to complete questionnaire regarding the usability of QuantonCal.

$>$ Laboratory calprotectin $(\mathrm{LC})$ readings $>250 \mu \mathrm{g} / \mathrm{L}$ as gold standard

\section{Figure 1: Correlation of home calprotectin values to laboratory calprotectin}

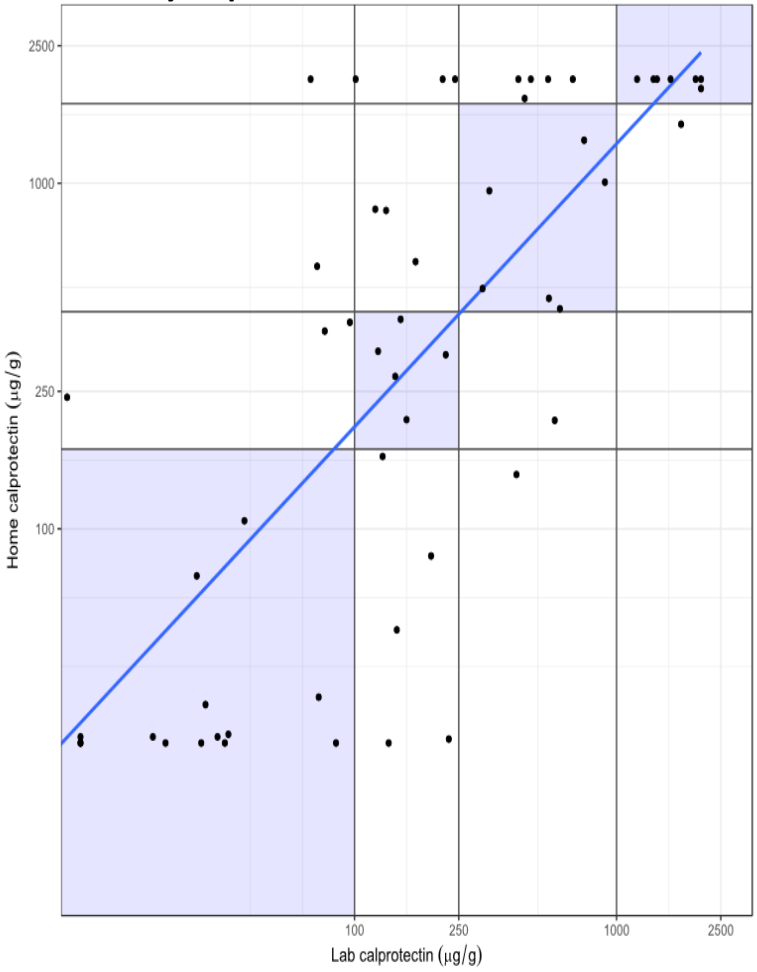

\section{Results}

59 patients: $32 \mathrm{CD}, 23 \mathrm{UC}, 4 \mathrm{IBD}-\mathrm{U}$.

> 38 (64\%) submitted at least one sample for analysis, 26 (44\%) submitted two samples. Three QuantonCal samples were excluded for invalid readings.

$>$ QuantonCal and LC readings significantly correlated; Spearman's rank correlation coefficient $=0.737, p<0.005$ (figure 1 ), however QuantonCal overestimated the LC value by about $70 \%$.

$>$ Median QuantonCal FC was $385 \mu \mathrm{g} / \mathrm{g}$ [IQR 31 - 1850], median laboratory FC was $148 \mu \mathrm{g} / \mathrm{g}$ [IQR 46 - 529]

$>$ QuantonCal test performance: area under the curve $(A \cup C)=0.870$ (95\% confidence interval $(\mathrm{Cl})=0.779-0.961)$, sensitivity $90 \%$, specificity $78 \%$, positive predictive value (PPV) $70 \%$, and negative predictive value (NPV) $94 \%$.

$>$ Of 26 patients that submitted two sets of samples, the change in calprotectin was compared between readings. Of 26 participants, 7 (27\%) where the laboratory calprotectin reading rose, the QuantonCal value fell, or vice versa (figure 2 ).

$>24$ patients completed the satisfaction questionnaire. Acceptability was high: 15 (63\%) thought the QuantonCal application was 'very easy', 7 (29\%) 'easy', . No patients reported the application was 'difficult' or 'very difficult' to use.

$>$ Preference towards QuantonCal compared to laboratory test: equal preference $9(37 \%)$, slight preference for iPhone 7 (29\%) and strong preference for iPhone 4 (17\%)

Figure 2: Serial calprotectin values: QuantonCal against LC Note values inside pink boxes have disparate results

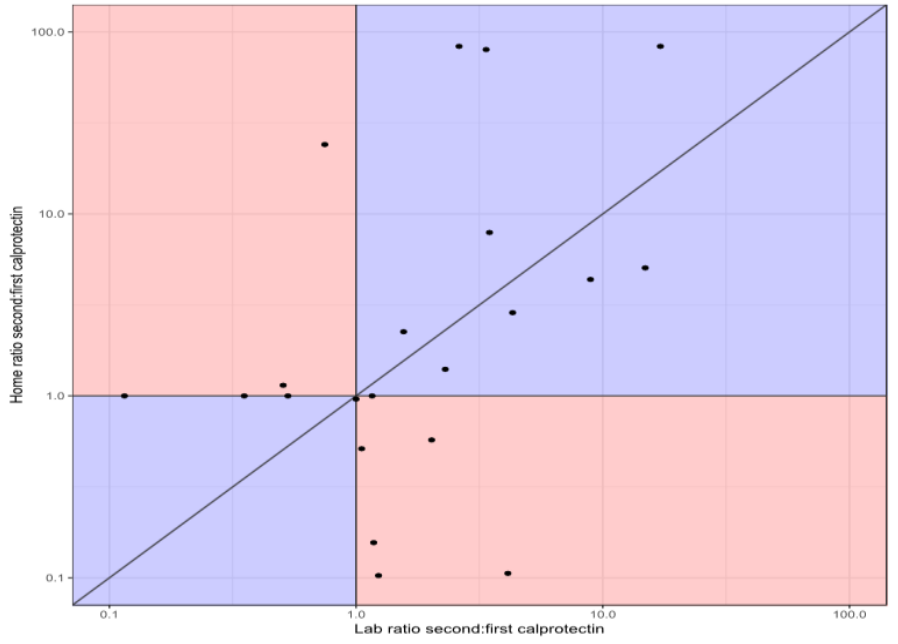

\section{Conclusions}

QuantonCal shows promise with values that correlate to LC. However, $27 \%$ of serial readings had disparate results. Clinically, this would either lead to delayed treatment, or inappropriate escalation of therapy. Patient acceptability remains high with slight preferences towards home testing. This study assumes LC as the gold standard but future studies could use endoscopy for comparison. 